\title{
Neurological outcomes following intraprocedural rerupture during coil embolization of ruptured intracranial aneurysms
}

\author{
Christopher J. Stapleton, MD, ${ }^{1}$ Brian P. Walcott, MD, ${ }^{1}$ William E. Butler, MD, ${ }^{1}$ \\ and Christopher S. Ogilvy, MD2,3
}

\begin{abstract}
1'Department of Neurosurgery, Massachusetts General Hospital and Harvard Medical School; '2Division of Neurosurgery, Department of Surgery, Beth Israel Deaconess Medical Center and Harvard Medical School; and ${ }^{3}$ Beth Israel Deaconess Medical Center Brain Aneurysm Institute, Boston, Massachusetts
\end{abstract}

\begin{abstract}
OBJECT Intraprocedural rerupture (IPR) of intracranial aneurysms during coil embolization is associated with significant periprocedural disability and death. However, whether this morbidity and mortality are secondary to an increased risk of vasospasm and hydrocephalus is unknown. The authors undertook this study to determine the in-hospital and long-term neurological outcomes for patients with aneurysmal subarachnoid hemorrhage (SAH) treated with coil embolization who suffer aneurysm rerupture during treatment.

METHODS The records of 156 patients admitted with SAH from previously untreated, ruptured, intracranial aneurysms and treated with endovascular coiling between January 2007 and January 2014 were retrospectively reviewed. Twelve patients (7.7\%) experienced IPR during coil embolization.

RESULTS Compared with the cohort of patients with uncomplicated coil embolization procedures, patients with aneurysm rerupture were more likely to require external ventricular drain (EVD) placement $(91.7 \%$ vs $58.3 \%, p=0.02)$ and postprocedural EVD placement ( $36.4 \%$ vs $7.1 \%, p=0.01$ ), to undergo permanent ventriculoperitoneal shunt placement $(50.0 \%$ vs $18.8 \%, p=0.02)$, to develop symptomatic vasospasm $(50.0 \%$ vs $18.1 \%, p=0.02)$, and to have longer lengths of hospital stay (median 21.5 days vs 15.0 days, $p=0.04$ ). Admission Hunt and Hess, modified Fisher, and Barrow Neurological Institute grades did not differ between the 2 cohorts, nor did long-term functional neurological outcomes as assessed by the modified Rankin Scale.
\end{abstract}

CONCLUSIONS Intraprocedural rerupture during coil embolization for ruptured intracranial aneurysms is associated with an increased risk of symptomatic vasospasm and need for temporary and permanent cerebrospinal fluid diversion for hydrocephalus.

http://thejns.org/doi/abs/10.3171/2014.9.JNS14616

KEY WORDS aneurysm; coil embolization; endovascular neurosurgery; hydrocephalus; intraprocedural rupture; subarachnoid hemorrhage; vasospasm; vascular disorders

$\mathrm{E}$ NDOVASCULAR coil embolization of ruptured intracranial aneurysms is an accepted treatment alternative to microsurgical clip obliteration. Since publication of the International Subarachnoid Aneurysm Trial (ISAT) and the Barrow Ruptured Aneurysm Trial (BRAT), coil embolization has become the dominant treatment modality for aneurysmal subarachnoid hemorrhage (aSAH) in many modern neurosurgical and neurointerventional practices. ${ }^{1,15,16}$ In fact, a recent analysis of the Nationwide Inpatient Sample reported that $54 \%$ to $69 \%$ of all ruptured intracranial aneurysms across all patient age groups were treated with coil embolization in 2009. ${ }^{1}$

The Cerebral Aneurysm Rerupture After Treatment (CARAT) study evaluated rates of rehemorrhage and intraprocedural rerupture (IPR) following both endovascular coil embolization and microsurgical clip obliteration for

ABBREVIATIONS aSAH = aneurysmal SAH; BNI = Barrow Neurological Institute; BRAT = Barrow Ruptured Aneurysm Trial; CARAT = Cerebral Aneurysm Rerupture After Treatment; EVD = external ventricular drain; ICP = intracranial pressure; ICU = intensive care unit; IOR = intraoperative aneurysm rupture; IPR = intraprocedural rerupture; ISAT = International Subarachnoid Aneurysm Trial; MGH = Massachusetts General Hospital; mRS = modified Rankin Scale; SAH = subarachnoid hemorrhage; SBP = systolic blood pressure; $T C D=$ transcranial Doppler; $V P=$ ventriculoperitoneal.

SUBMITTED March 18, 2014. ACCEPTED September 18, 2014.

INCLUDE WHEN CITING Published online October 31, 2014; DOI: 10.3171/2014.9.JNS14616.

DISCLOSURE The authors have no conflicts of interest, sources of financial support, or industry affiliations to disclose relevant to this investigation. 
ruptured intracranial aneurysms. ${ }^{4}$ While IPR was more common in the microsurgical cohort $(18.6 \%$ vs $5.4 \%)$, periprocedural death or disability was more common in patients who suffered IPR during coil embolization (63\% vs 31\%). ${ }^{4}$ Therefore, while the overall risk of an IPR event is lower with coil embolization, the consequences of this complication are more profound in this setting. Several retrospective studies and meta-analyses have examined risk factors for aneurysm rerupture during endovascular coiling, techniques for IPR management, and radiographic and neurological outcomes following IPR., 13,14,17,21,24 No study, however, has reported comparative rates of vasospasm or need for CSF diversion in patients who suffer IPR versus those who do not. The purpose of this study was to evaluate the effect of IPR during endovascular coil embolization of ruptured intracranial aneurysms with consequent rehemorrhage and contrast extravasation on vasospasm, requirement for temporary or permanent CSF diversion, and long-term functional neurological outcomes.

\section{Methods}

\section{Patients and Data Collection}

Following approval by the Massachusetts General Hospital (MGH) institutional review board, the records of 563 patients admitted to MGH with subarachnoid hemorrhage (SAH) between January 2007 and January 2014 were retrospectively reviewed. Among these 563 patients, 156 adults with SAH from a previously untreated, ruptured, saccular intracranial aneurysm treated with coil embolization were identified. Twelve of these patients experienced an IPR during treatment with documented contrast extravasation on postprocedural CT head imaging (Fig. 1A-D). The study sample was collected by reviewing the MGH cerebrovascular surgery and radiology databases for endovascularly treated ruptured aneurysms within the study period. All historical, clinical, radiographic, and follow-up information was obtained from the electronic medical record.

The following data were collected: age; gender; medical and social history; aneurysm location and size; Hunt and Hess grade; modified Fisher grade; ${ }^{5}$ Barrow Neurological Institute (BNI) grade; ${ }^{23}$ requirement for and length of temporary CSF diversion; intraprocedural complications and management during coil embolization; maximum transcranial Doppler (TCD) velocity in any vessel; maximum Lindegaard ratio; presence of vasospasm on CT, MR, or catheter angiography; presence of symptomatic vasospasm; requirement for endovascular intervention for vasospasm; requirement for VP shunting; and modified Rankin Scale (mRS) scores on admission, at 1 year, and at last follow-up.

Patients with follow-up mRS scores of 0-2 were considered to have a good clinical outcome from treatment. If the prehemorrhage mRS score was 3-5, a patient was also considered to have a good outcome from treatment if the mRS score remained the same at the time of last follow-up.

\section{Subarachnoid Hemorrhage Management}

All aneurysms were secured within 36 hours of pre-
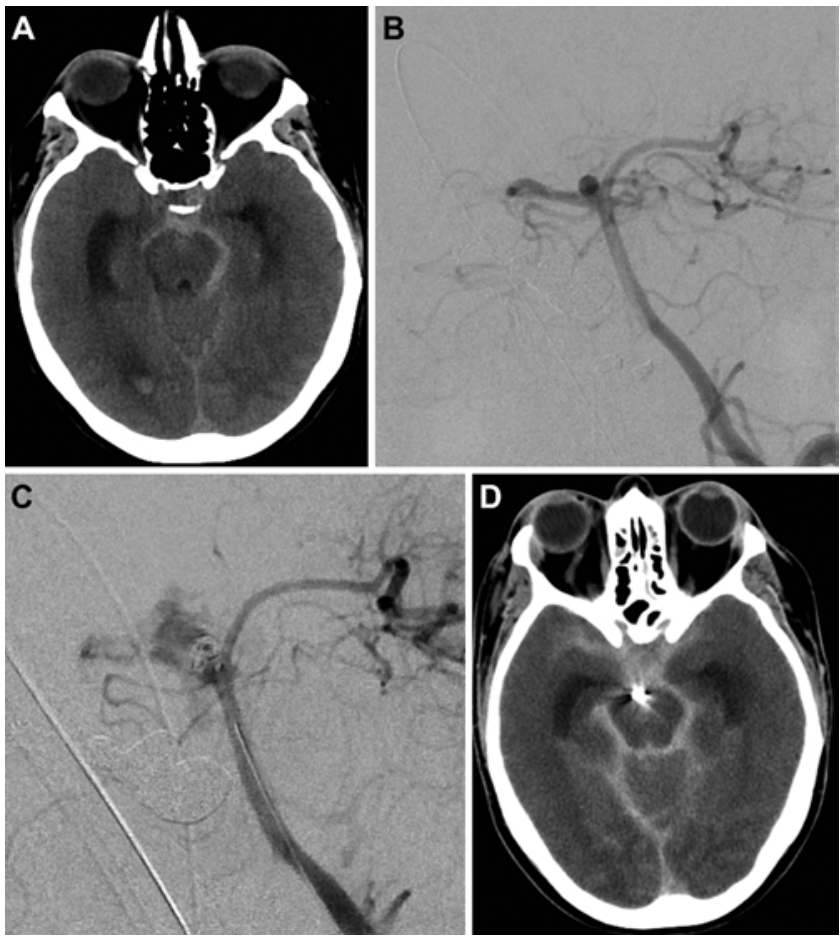

FIG. 1. A: Axial CT image showing SAH from a ruptured basilar apex aneurysm. B and C: Right anterior oblique catheter angiograms obtained in the same case showing a ruptured basilar apex aneurysm (B) and active contrast extravasation $(C)$ following intraprocedural rupture (IPR) during coil embolization. D: Axial CT image showing increased $\mathrm{SAH}$ and contrast following IPR of the ruptured basilar apex aneurysm.

sentation, and all patients were cared for in a dedicated neurosciences intensive care unit (ICU) according to a standardized SAH protocol. ${ }^{3}$ Patients with clinical or radiographic hydrocephalus or with admission Hunt and Hess grades $\geq$ III underwent external ventricular drain (EVD) placement for CSF diversion and intracranial pressure (ICP) monitoring. EVDs were managed according to a previously published protocol. ${ }^{11}$ Phenytoin or levetiracetam was administered until the aneurysm was secured and continuous electroencephalography demonstrated no seizure activity. All patients received oral nimodipine for vasospasm prevention and underwent daily TCD ultrasonography for vasospasm detection. CT or MR angiography was performed if patients had elevated or rising TCD values or demonstrated a change in neurological status thought to be attributable to vasospasm. Catheter angiography with or without pharmacological or mechanical intra-arterial vasodilation was performed in patients with CT or MR angiographic vasospasm or patients in whom there was a high clinical suspicion for vasospasm despite negative findings on CT or MR angiography. All patients with vasospasm received hypertensive and hypervolemic therapy prior to initiation of endovascular intervention.

\section{Vasospasm}

Vasospasm was defined as new or progressive intracranial arterial narrowing (mild, moderate, or severe) identified on catheter or CT angiography. ${ }^{20,23}$ Elevated TCD blood flow velocity measurements often prompted 
vascular imaging, but sonographic data were not used to define vasospasm. Symptomatic vasospasm was defined as neurological symptoms referable to a region of angiographic vasospasm in the absence of alternative explanations. The determination of symptomatic vasospasm was made during the patient's hospital stay in a nonblinded manner by the staff neurosurgeon (C.S.O.) and neurocritical care physician of record.

\section{Diagnosis and Management of IPR}

Intraprocedural rerupture was diagnosed by direct fluoroscopic visualization of contrast extravasation from the aneurysm sac with or without an endovascular device outside the limits of the aneurysm. If the balloon-assisted technique was used, the balloon was inflated immediately upon suspected aneurysm rerupture to seal the aneurysm neck. Coils were deployed rapidly to achieve dome protection. In select cases, heparin was reversed with the administration of protamine sulfate. If an EVD was in place, CSF was drained to maintain normal ICP. Mannitol or $23.4 \%$ hypertonic saline was administered for ICP control as necessary, and systolic blood pressures (SBPs) were maintained at less than $140 \mathrm{~mm} \mathrm{Hg}$ during the rerupture period. Postprocedural noncontrast CT imaging was obtained in all cases. An EVD was placed as necessary for hydrocephalus or poor neurological status.

\section{Statistical Analysis}

Descriptive statistics were calculated for clinical and radiographic factors, using the median as a measure of central tendency. A univariate analysis of clinical characteristics and outcomes was performed. Comparisons of continuous variables with nonnormal distributions were made using the nonparametric Mann-Whitney U-test. Contingency statistics on categorical variables were performed with the Fisher exact test. All statistical tests were two-sided and $p<0.05$ was prospectively determined to establish statistical significance. All analyses were performed using GraphPad version 6 (Prism).

\section{Results}

\section{Patient Demographics}

One hundred fifty-six patients were analyzed, 12 of whom experienced an IPR event (7.7\%) during treatment. All patients had previously untreated, ruptured, saccular aneurysms. Table 1 summarizes the demographic details of this cohort. The median age was 60.2 years, and $72.4 \%$ of the cohort was female. A history of smoking and hypertension was confirmed in $69(44.2 \%)$ and 72 (46.2\%) patients, respectively. Prehemorrhage mRS classifications demonstrated a significantly greater proportion of patients with mRS 0 in the IPR cohort $(91.7 \%$ vs $60.4 \%$, p $=0.03$ ). However, the two cohorts were similar when patients were stratified by clinical grade $(\mathrm{mRS} 0-2=$ good, $3-5=$ fair/poor) prior to SAH (91.7\% [IPR] vs $94.4 \%$ [no IPR], $\mathrm{p}=0.52$ ). The median length of follow-up for the entire cohort was 16.1 months. Length of follow-up did not differ significantly between the study groups.

\section{Subarachnoid Hemorrhage Characteristics}

Table 1 summarizes the distribution of admission Hunt and Hess, modified Fisher, and BNI grades in addition to aneurysm location and size and need for CSF diversion among patients in the study cohort. Admission Hunt and Hess grade classifications showed a significantly greater proportion of patients with a Grade II status in the IPR cohort ( $50 \%$ vs $18.8 \%, \mathrm{p}=0.02)$; however, the two groups were similar when patients were classified by clinical grade (Hunt and Hess Grade I-II = good; III-V = fair/ poor) upon admission (58.3\% [IPR] vs $47.9 \%$ [no IPR], p $=0.56$ ). Modified Fisher and BNI grades on presentation were similar across all grades between those who suffered an IPR and those who did not.

The most common location for aneurysms treated with coil embolization in this series included the anterior communicating artery (41.7\%), posterior communicating artery $(23.7 \%)$, and basilar artery apex $(14.7 \%)$. The median aneurysm size was $6.0 \mathrm{~mm}$. There were no significant differences in aneurysm location or size between the two study groups.

EVDs were placed in a total of 95 patients (60.9\%), with a greater proportion of patients with an IPR requiring CSF diversion and ICP management (91.7\% vs 58.3\%, $\mathrm{p}=0.02)$. Similarly, postprocedural EVDs were placed more frequently in the IPR cohort $(36.4 \%$ vs $7.1 \%, \mathrm{p}=$ $0.01)$. There was no difference in the overall length of EVD use between the two cohorts.

\section{Intraprocedural Rerupture Management}

Table 2 summarizes the details of the IPR events. One aneurysm rupture occurred during guidewire and microcatheter navigation, while the other 11 ruptures occurred during coil deployment. The balloon-assisted technique was used in 2 cases. In 6 cases, a hemodynamic response (elevated ICP and/or elevated SBP) was observed. To prevent continued aneurysm rupture, protamine sulfate was given for heparin reversal in 2 cases, while additional coils were rapidly deployed for dome protection in all cases. On postprocedural CT imaging, the pattern of new hemorrhage and/or contrast extravasation was classified as focal in 2 cases and diffuse in 10 cases.

\section{In-Hospital and Long-Term Outcomes}

Similar to the need for temporary ventricular drainage, a significantly greater proportion of patients in the IPR cohort required VP shunt placement for permanent CSF diversion due to hydrocephalus $(50 \%$ vs $18.8 \%$, $\mathrm{p}=$ 0.02 ). While there was a trend toward a greater incidence of angiographic vasospasm in the IPR cohort (58.3\% vs $32.6 \%)$, the difference was not statistically significant ( $\mathrm{p}=$ 0.10 ). There was no difference in the rate of endovascular treatment for vasospasm between the two groups. Compared with those patients who did not suffer IPR during aneurysm treatment, patients with an IPR event experienced symptomatic vasospasm postprocedurally at a higher rate $(50 \%$ vs $18.1 \%, \mathrm{p}=0.02)$. In addition, patients in the IPR group had a median length of stay of 21.5 days, while those without an IPR had a median length of stay of 15.0 days $(p=0.04)$. The rate of in-hospital SAH-asso- 
TABLE 1. Characteristics of 156 patients treated with endovascular coil embolization for ruptured intracranial aneurysms*

\begin{tabular}{|c|c|c|c|}
\hline Characteristic & No IPR & IPR & $p$ Value \\
\hline Total no. of patients & 144 & 12 & - \\
\hline Median age (yrs) & 60.4 & 56.9 & 0.57 \\
\hline Female sex & $103(71.5)$ & $10(83.3)$ & 0.52 \\
\hline History of smoking & $64(44.4)$ & $5(41.6)$ & 0.72 \\
\hline History of hypertension & $66(45.8)$ & $6(50.0)$ & $>0.99$ \\
\hline \multicolumn{4}{|l|}{ Prehemorrhage mRS } \\
\hline 0 & $87(60.4)$ & $11(91.7)$ & 0.03 \\
\hline 1 & $25(17.4)$ & 0 & 0.22 \\
\hline 2 & $24(16.7)$ & 0 & 0.22 \\
\hline 3 & $7(4.9)$ & $1(8.3)$ & 0.48 \\
\hline 4 & $1(0.69)$ & 0 & $>0.99$ \\
\hline 5 & 0 & 0 & $>0.99$ \\
\hline $0-2$ & $136(94.4)$ & $11(91.7)$ & 0.52 \\
\hline \multicolumn{4}{|l|}{ Aneurysm location } \\
\hline OphA or SHA & $11(7.6)$ & $1(8.3)$ & $>0.99$ \\
\hline PCoA & $34(23.6)$ & $3(25.0)$ & $>0.99$ \\
\hline Anterior choroidal a. & $3(2.1)$ & 0 & $>0.99$ \\
\hline ICA terminus & $1(0.69)$ & 0 & $>0.99$ \\
\hline $\mathrm{ACoA}$ & $59(40.9)$ & $6(50.0)$ & 0.56 \\
\hline Pericallosal a. & $1(0.69)$ & 0 & $>0.99$ \\
\hline Anterior temporal a. & $1(0.69)$ & 0 & $>0.99$ \\
\hline MCA & $1(0.69)$ & 0 & $>0.99$ \\
\hline Basilar apex & $21(14.6)$ & $2(16.7)$ & 0.69 \\
\hline PCA & $4(2.8)$ & 0 & $>0.99$ \\
\hline SCA & $3(2.1)$ & 0 & $>0.99$ \\
\hline PICA & $5(3.5)$ & 0 & $>0.99$ \\
\hline Median aneurysm size (mm) & 6.0 & 6.5 & 0.99 \\
\hline \multicolumn{4}{|l|}{ Hunt \& Hess grade } \\
\hline I & $42(29.2)$ & $1(8.3)$ & 0.18 \\
\hline$\|$ & $27(18.8)$ & $6(50.0)$ & 0.02 \\
\hline III & $44(30.6)$ & $4(33.3)$ & $>0.99$ \\
\hline IV & $23(15.9)$ & $1(8.3)$ & 0.69 \\
\hline V & $8(5.6)$ & 0 & $>0.99$ \\
\hline$|-| \mid$ & $69(47.9)$ & $7(58.3)$ & 0.56 \\
\hline \multicolumn{4}{|l|}{ Modified Fisher grade } \\
\hline 0 & $6(4.2)$ & 0 & $>0.99$ \\
\hline 1 & $20(13.9)$ & $1(8.3)$ & $>0.99$ \\
\hline 2 & $28(19.4)$ & $2(16.7)$ & $>0.99$ \\
\hline 3 & $26(18.1)$ & $5(41.7)$ & 0.06 \\
\hline 4 & $64(44.4)$ & $4(33.3)$ & 0.55 \\
\hline \multicolumn{4}{|l|}{ BNI grade } \\
\hline 1 & $8(5.6)$ & 0 & $>0.99$ \\
\hline 2 & $49(34.0)$ & $5(41.7)$ & 0.75 \\
\hline 3 & $59(40.9)$ & $4(33.3)$ & 0.76 \\
\hline 4 & $21(14.6)$ & $2(16.7)$ & 0.69 \\
\hline 5 & $7(4.9)$ & $1(8.3)$ & 0.48 \\
\hline EVD & $84(58.3)$ & $11(91.7)$ & 0.02 \\
\hline Postprocedural placement & $6(7.1)$ & $4(36.4)$ & 0.01 \\
\hline
\end{tabular}


TABLE 1. Characteristics of 156 patients treated with endovascular coil embolization for ruptured intracranial aneurysms* (continued)

\begin{tabular}{lccc}
\hline Characteristic & No IPR & IPR & p Value \\
\hline Median duration of EVD use (days) & 14.0 & 15.0 & 0.21 \\
\hline a. = artery; ACOA = anterior communicating artery; ICA = internal carotid artery; IPR = intraprocedural rerupture; MCA = middle \\
cerebral artery; OphA = ophthalmic artery; PCA = posterior cerebral artery; PCoA = posterior communicating artery; PICA = pos- \\
terior inferior cerebellar artery; SCA = superior cerebellar artery; SHA = superior hypophyseal artery. \\
$*$ Values represent number of patients (\%) unless otherwise indicated. Boldface type indicates statistical significance.
\end{tabular}

ciated mortality was $8.3 \%$ in the group with an IPR and $10.4 \%$ in the group without a rerupture event $(\mathrm{p}>0.99)$. Despite the statistically significant differences in in-hospital outcomes, there were no significant differences in the distribution of $\mathrm{mRS}$ scores or clinical grades between the 2 cohorts at the time of last follow-up. Table 3 lists the in-hospital and long-term outcomes for all patients.

Seven (58.3\%) of 12 patients who suffered an IPR event were classified as Hunt and Hess Grade I or II on admission. Following aneurysm rerupture during treatment, the Hunt and Hess grade remained I or II in only $2(16.7 \%)$ of these 12 patients $(\mathrm{p}=0.04)$, indicating a significant decline in neurological status following IPR. Figure 2 illustrates the distribution of Hunt and Hess grades before and after coil embolization with IPR.

\section{Discussion}

Since the introduction of Guglielmi detachable coils (GDCs) to clinical practice in 1995, endovascular coil embolization has emerged as the predominant treatment modality for most ruptured intracranial aneurysms. Analysis of the Nationwide Inpatient Sample demonstrates an increase in the frequency of coil embolization for aSAH from $7 \%-22 \%$ in 2001 to $54 \%-69 \%$ in 2009 across all age categories. ${ }^{1}$ This upward trend is largely due to the reports of ISAT and BRAT, which showed that 1 year after treatment there were $6.9 \%$ and $13.5 \%$, respectively, fewer dependent or dead patients in the cohorts treated with endovascular coiling as opposed to microsurgical clipping for ruptured aneurysms. ${ }^{1,15,16}$ Given the increased frequen-

TABLE 2. Characteristics and management of IPR during endovascular coil embolization for ruptured intracranial aneurysms in 12 patients

\begin{tabular}{|c|c|}
\hline Characteristic & No. (\%) \\
\hline \multicolumn{2}{|l|}{ Timing of perforation } \\
\hline Guidewire \& microcatheter navigation & $1(8.3)$ \\
\hline Balloon catheter navigation & 0 \\
\hline Coil placement & $11(91.7)$ \\
\hline Balloon-assisted embolization & $2(16.7)$ \\
\hline Hemodynamic response to IPR & $6(50.0)$ \\
\hline \multicolumn{2}{|l|}{ Management of IPR } \\
\hline Protamine sulfate for heparin reversal & $2(16.7)$ \\
\hline Additional coils & $12(100.0)$ \\
\hline \multicolumn{2}{|c|}{ Pattern of hemorrhage \&/or contrast extravasation } \\
\hline Focal & $2(16.7)$ \\
\hline Diffuse & $10(83.3)$ \\
\hline
\end{tabular}

cy of coil embolization in modern practice, a detailed understanding of potential complications and their consequences is necessary. Overall, aneurysm rerupture during endovascular treatment is an uncommon event, with both CARAT and ISAT reporting a 5.4\% IPR rate and Cloft and Kallmes reporting a $4.1 \%$ rerupture rate. ${ }^{2,4,16}$ Despite its low occurrence, the likelihood of periprocedural death or disability has been documented to be as high as $63 \% .^{2,4}$ In this report, we present a cohort of 156 patients with aSAH treated with coil embolization, and demonstrate an increase in rates of symptomatic vasospasm, requirement for temporary and permanent CSF diversion, and overall length of hospital stay in those patients who suffer aneurysm rerupture with contrast extravasation during endovascular treatment.

Vasospasm and hydrocephalus are significant causes of morbidity and mortality following aSAH, with $20 \%-$ $30 \%$ of patients developing symptomatic vasospasm and approximately $10 \%$ of patients requiring VP shunt placement for treatment of hydrocephalus. ${ }^{8,22}$ Outcomes in our cohort of uncomplicated coil embolizations for aSAH parallel these national data. However, for patients in whom an IPR occurred, rates of symptomatic vasospasm and EVD and VP shunt placement were significantly increased. Although numerous studies have analyzed the complication of IPR during coil embolization of ruptured intracranial aneurysms, ${ }^{2}$ this is the first report in the neurosurgical literature to demonstrate an increased risk of symptomatic vasospasm and hydrocephalus in patients who experience IPR.

While the mechanistic underpinnings of vasospasm are incompletely understood, it is well appreciated that the risk of vasospasm directly correlates with the volume and distribution of SAH, as clot lysis and erythrocyte degradation products can induce large-vessel vasoconstriction and microvascular dysfunction, increase inflammation and oxidative stress, and promote blood-brain barrier dysfunction. ${ }^{5,12,22,23}$ Therefore, our observation of an increased risk of symptomatic vasospasm following IPR may simply relate to the greater burden of SAH and contrast material in these patients. In contrast to our observations with respect to symptomatic vasospasm, we observed only a trend toward a greater rate of angiographic vasospasm in those patients who suffer rerupture events, but the difference did not reach statistical significance, possibly due to our small sample size. Interestingly, of the 47 patients who developed angiographic vasospasm in the cohort without an IPR, only 26 (55.3\%) were symptomatic, whereas $6(85.7 \%)$ of the 7 patients in the IPR group with angiographic vasospasm eventually developed referable symptoms. Brain injury is known to 
TABLE 3. Outcomes following endovascular coil embolization for ruptured intracranial aneurysms in 156 patients*

\begin{tabular}{lccc}
\hline \multicolumn{1}{c}{ Outcome } & No IPR & IPR & p Value \\
\hline VP shunt & $27(18.8)$ & $6(50.0)$ & 0.02 \\
\hline Angiographic vasospasm & $47(32.6)$ & $7(58.3)$ & 0.10 \\
\hline Endovascular intervention for angiographic vasospasm & $25(53.2)$ & $4(57.1)$ & $>0.99$ \\
\hline Symptomatic vasospasm & $26(18.1)$ & $6(50.0)$ & 0.02 \\
\hline Median LOS (days) & 15.0 & 21.5 & 0.04 \\
\hline In-hospital mortality & $15(10.4)$ & $1(8.3)$ & $>0.99$ \\
\hline Median length of follow-up (mos) & 8.4 & 23.5 & 0.45 \\
\hline Follow-up mRS score & & $4(33.3)$ & 0.74 \\
\hline 0 & $38(26.4)$ & $1(8.3)$ & 0.47 \\
\hline 1 & $29(20.1)$ & $2(16.7)$ & $>0.99$ \\
\hline 2 & $24(16.7)$ & $1(8.3)$ & $>0.99$ \\
\hline 3 & $19(13.2)$ & $2(16.7)$ & 0.20 \\
\hline 4 & $9(6.3)$ & $1(8.3)$ & 0.28 \\
\hline 5 & $3(2.1)$ & $1(8.3)$ & $>0.99$ \\
\hline 6 & $22(15.3)$ & $5(41.7)$ & 0.76 \\
\hline Clinical outcome & $53(36.8)$ & & 0.54 \\
\hline Good & & $7(58.3)$ & 0.54 \\
\hline Poor/death & $97(67.4)$ & $5(41.7)$ & \\
\hline VaS length of hospital stay. & $47(32.6)$ & & \\
\hline
\end{tabular}

occur immediately after aneurysm rupture via impaired cerebral blood flow with associated cerebral ischemia. ${ }^{19}$ Accordingly, homeostatic mechanisms that regulate ICP and cerebral blood flow are compromised in aSAH, and this dysregulation is likely magnified following rerupture events, such that patients with an IPR who have angiographic vasospasm may be more prone to develop referable neurological symptoms from this vasospasm. This same phenomenon is not observed during intraoperative aneurysm rupture (IOR) during microsurgical clipping. Sheth et al. reviewed 75 IORs in 500 patients at the University of California, San Francisco, and demonstrated

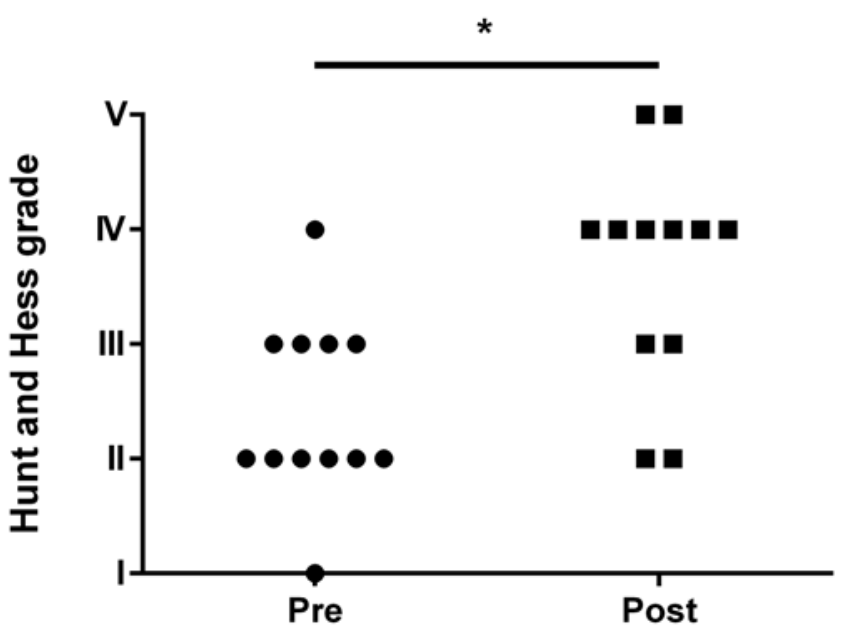

FIG. 2. Distribution of Hunt and Hess grades obtained before and after coil embolization with IPR of ruptured intracranial aneurysms in 12 patients. ${ }^{*} p=0.04$. no difference between patients with or without an IOR in the rates of either angiographic vasospasm $(66 \%$ vs $65 \%$, respectively) or symptomatic vasospasm ( $36 \%$ vs $41 \%$, respectively). ${ }^{20}$ Following an IOR, the blood is cleared from the operative field by irrigation and suction, and patients are, therefore, not often subjected to an increased amount of SAH postoperatively. In addition, during a craniotomy, the intracranial compartment is no longer a closed space. Thus, when an aneurysm reruptures during clipping, patients do not have the same acute rise in ICP that patients who suffer IPR experience.

Following aSAH, hydrocephalus is reported to occur in $20 \%$ to $30 \%$ of patients, with approximately $10 \%$ of patients eventually requiring VP shunt placement for permanent CSF diversion, irrespective of treatment modality. ${ }^{6,8}$ While the exact mechanism by which hydrocephalus develops after aSAH is poorly understood, it is generally thought that fibrosis of the arachnoid granulations leads to impaired CSF absorption with consequent communicating hydrocephalus. The Cooperative Aneurysm Study reported several risk factors for the development of symptomatic hydrocephalus, ${ }^{7}$ with admission neurological status, radiographic hydrocephalus, increased $\mathrm{SAH}$, and vasospasm being several significant factors. While admission Hunt and Hess, modified Fisher, and BNI grades did not differ between our study cohorts, all patients with a rerupture event had a significant increase in their SAH burden, mostly in a diffuse distribution. Accordingly, 11 (91.7\%) of 12 patients with an IPR required placement of an EVD, and 6 of these 11 patients required a permanent shunt.

CARAT reported periprocedural death and disability in $63 \%$ of patients, and Cloft and Kallmes reported a $33 \%$ risk of death and 5\% risk of disability in patients 
following IPR during coil embolization, but few studies have examined long-term outcomes in this patient population. ${ }^{2,4}$ Zhang et al. followed 12 patients for a median of 14 months after IPR and noted complete recovery in 7 patients and 1 death. ${ }^{24}$ Similarly, Luo et al. followed 10 patients for a median of 20.5 months and noted good recoveries (mRS score $\leq 2)$ in 6 patients, moderate disability in 1 patient (mRS score 4), and 3 deaths. ${ }^{14}$ At a median of 4.5 months, Levy et al. found 4 of 6 patients to be free of disability or dependence and 2 patients had died. ${ }^{13}$ In our series, at a median of 23.5 months of follow-up, 7 of 12 patients were free of either disability or dependence (mRS score $\leq 2$ ), 4 patients had moderate disability (mRS score 3-5), and 1 patient had died during hospitalization. In effect, the risk of death or dependency following an IPR, therefore, in this series was $41.7 \%$, compared with $36.8 \%$ in the group of patients with an uncomplicated coil embolization procedure. While a significant portion of patients did experience an acute neurological decline following IPR, as evidenced by the difference in Hunt and Hess grades before and after endovascular coiling, not all of these patients went on to have a poor clinical outcome. The reason for this is likely multifold. Prompt recognition of IPR by the neurosurgeon, neurointerventionalist, and neuroanesthesiologist can result in the implementation of immediate corrective actions to reduce continued rupture, including coil deployment, SBP control, and heparin reversal. In addition, 7 of 12 patients had an EVD in place during IPR, allowing for a rapid normalization of ICP and a reduction in secondary brain injury. Finally, advancements in SAH-specific neurocritical care strategies and vasospasm detection and management have allowed focused interventions to improve patient outcomes following aSAH with or without IPR. ${ }^{9}$

An additional aspect of IPR that must be considered when analyzing the risk of vasospasm and need for CSF diversion is the presence of contrast material in the subarachnoid space. All cerebral angiography in our study was performed with an iopromide contrast agent (Ultravist, Bayer HealthCare LLC). To our knowledge, the effect of radiographic dyes within the subarachnoid space on the cerebral vasculature and CSF absorption mechanisms has not been studied. Karstoft et al., however, demonstrated that rabbit coronary arteries undergo segmental vasoconstriction when exposed to iodinated contrast media. ${ }^{10}$ As cerebral arteries lack a robust adventitial layer and external elastic lamina ${ }^{18}$ it is possible that the cerebral vasculature may be exquisitely sensitive to contrast agents. Moreover, like blood, contrast material is more viscous than CSF, and its presence in high concentrations in the subarachnoid space may reduce CSF resorption through the arachnoid villi, leading to hydrocephalus.

Our study has the limitations inherent in any single-institution, retrospective series. Compared with the number of total admissions for aSAH, the number of patients with IPR in our case series is quite small. Therefore, while several comparisons in our study met statistical significance, such observations should be validated in larger populations and across multiple high-volume centers. In addition, while the difference was not statistically significant, patients with an IPR had greater than twice the median length of follow-up. As a result, patients with aneurysm rerupture were allowed more time to "recover" than patients with uncomplicated coil procedures, perhaps explaining the lack of any differences in $\mathrm{mRS}$ classifications between the 2 groups. Finally, in our series, patients were only considered to have an IPR if there was observable contrast extravasation intraprocedurally or on postprocedural CT head imaging. Cases in which patients were noted to have an endovascular device outside the confines of the aneurysm but did not have a noted hemodynamic response intraprocedurally nor the presence of contrast in the subarachnoid space on postprocedural CT imaging were analyzed as having had no IPR event.

\section{Conclusions}

Aneurysm rerupture during coil embolization is a rare complication, but its occurrence is associated with an increased risk of symptomatic vasospasm and need for temporary and permanent CSF diversion. Thus, the presence of an IPR event should alert the treating neurosurgeon, neurointerventionalist, and neurocritical care physician to these potential complications, which may trigger early, focused therapies to reduce morbidity and mortality. In this series, long-term functional outcomes did not differ between patients with aneurysm rerupture during treatment and those with uncomplicated coil embolization procedures. To validate our observations, a study with larger sample sizes incorporating patients across numerous high-volume centers should be conducted.

\section{References}

1. Brinjikji W, Lanzino G, Rabinstein AA, Kallmes DF, Cloft HJ: Age-related trends in the treatment and outcomes of ruptured cerebral aneurysms: a study of the nationwide inpatient sample 2001-2009. AJNR Am J Neuroradiol 34:1022-1027, 2013

2. Cloft HJ, Kallmes DF: Cerebral aneurysm perforations complicating therapy with Guglielmi detachable coils: a metaanalysis. AJNR Am J Neuroradiol 23:1706-1709, 2002

3. Connolly ES Jr, Rabinstein AA, Carhuapoma JR, Derdeyn CP, Dion J, Higashida RT, et al: Guidelines for the management of aneurysmal subarachnoid hemorrhage: a guideline for healthcare professionals from the American Heart Association/American Stroke Association. Stroke 43:1711-1737, 2012

4. Elijovich L, Higashida RT, Lawton MT, Duckwiler G, Giannotta S, Johnston SC: Predictors and outcomes of intraprocedural rupture in patients treated for ruptured intracranial aneurysms: the CARAT study. Stroke 39:1501-1506, 2008

5. Frontera JA, Claassen J, Schmidt JM, Wartenberg KE, Temes R, Connolly ES Jr, et al: Prediction of symptomatic vasospasm after subarachnoid hemorrhage: the modified Fisher scale. Neurosurgery 59:21-27, 2006

6. Germanwala AV, Huang J, Tamargo RJ: Hydrocephalus after aneurysmal subarachnoid hemorrhage. Neurosurg Clin $\mathbf{N}$ Am 21:263-270, 2010

7. Graff-Radford NR, Torner J, Adams HP Jr, Kassell NF: Factors associated with hydrocephalus after subarachnoid hemorrhage. A report of the Cooperative Aneurysm Study. Arch Neurol 46:744-752, 1989

8. Hoh BL, Kleinhenz DT, Chi YY, Mocco J, Barker FG II: Incidence of ventricular shunt placement for hydrocephalus with clipping versus coiling for ruptured and unruptured cerebral aneurysms in the Nationwide Inpatient Sample database: 2002 to 2007. World Neurosurg 76:548-554, 2011 
9. Josephson SA, Douglas VC, Lawton MT, English JD, Smith WS, Ko NU: Improvement in intensive care unit outcomes in patients with subarachnoid hemorrhage after initiation of neurointensivist co-management. Clinical article. J Neurosurg 112:626-630, 2010

10. Karstoft J, Bääth L, Jansen I, Edvinsson L: Contrast mediuminduced vasoconstrictions. An investigation of the vasoconstrictive action of iohexol in isolated rabbit coronary arteries. Acta Radiol 36:198-203, 1995

11. Klopfenstein JD, Kim LJ, Feiz-Erfan I, Hott JS, Goslar P, Zabramski JM, et al: Comparison of rapid and gradual weaning from external ventricular drainage in patients with aneurysmal subarachnoid hemorrhage: a prospective randomized trial. J Neurosurg 100:225-229, 2004

12. Kolias AG, Sen J, Belli A: Pathogenesis of cerebral vasospasm following aneurysmal subarachnoid hemorrhage: putative mechanisms and novel approaches. J Neurosci Res 87:1-11, 2009

13. Levy E, Koebbe CJ, Horowitz MB, Jungreis CA, Pride GL, Dutton K, et al: Rupture of intracranial aneurysms during endovascular coiling: management and outcomes. Neurosurgery 49:807-813, 2001

14. Luo CB, Mu-Huo Teng M, Chang FC, Lin CJ, Guo WY, Chang CY: Intraprocedure aneurysm rupture in embolization: clinical outcome with imaging correlation. J Chin Med Assoc 75:281-285, 2012

15. McDougall CG, Spetzler RF, Zabramski JM, Partovi S, Hills NK, Nakaji P, et al: The Barrow Ruptured Aneurysm Trial. Clinical article. J Neurosurg 116:135-144, 2012

16. Molyneux A, Kerr R, Stratton I, Sandercock P, Clarke M, Shrimpton J, et al: International Subarachnoid Aneurysm Trial (ISAT) of neurosurgical clipping versus endovascular coiling in 2143 patients with ruptured intracranial aneurysms: a randomised trial. Lancet 360:1267-1274, 2002

17. Santillan A, Gobin YP, Greenberg ED, Leng LZ, Riina HA, Stieg PE, et al: Intraprocedural aneurysmal rupture during coil embolization of brain aneurysms: role of balloon-assisted coiling. AJNR Am J Neuroradiol 33:2017-2021, 2012

18. Sehba FA, Bederson JB: Mechanisms of acute brain injury after subarachnoid hemorrhage. Neurol Res 28:381-398, 2006

19. Sehba FA, Hou J, Pluta RM, Zhang JH: The importance of early brain injury after subarachnoid hemorrhage. Prog Neurobiol 97:14-37, 2012

20. Sheth SA, Hausrath D, Numis AL, Lawton MT, Josephson SA: Intraoperative rerupture during surgical treatment of aneurysmal subarachnoid hemorrhage is not associated with an increased risk of vasospasm. Clinical article. J Neurosurg 120:409-414, 2014

21. Tummala RP, Chu RM, Madison MT, Myers M, Tubman D, Nussbaum ES: Outcomes after aneurysm rupture during endovascular coil embolization. Neurosurgery 49:1059-1067, 2001

22. Velat GJ, Kimball MM, Mocco JD, Hoh BL: Vasospasm after aneurysmal subarachnoid hemorrhage: review of randomized controlled trials and meta-analyses in the literature. World Neurosurg 76:446-454, 2011

23. Wilson DA, Nakaji P, Abla AA, Uschold TD, Fusco DJ, Oppenlander ME, et al: A simple and quantitative method to predict symptomatic vasospasm after subarachnoid hemorrhage based on computed tomography: beyond the Fisher scale. Neurosurgery 71:869-875, 2012

24. Zhang Y, Li G, Cai Y, Zhu J, Huang S, Li T, et al: Rupture during the endovascular treatment of intracranial aneurysms: outcomes and technical aspects. Acta Neurochir (Wien) 155:569-577, 2013

\section{Author Contributions}

Conception and design: Stapleton, Walcott. Acquisition of data: Stapleton, Walcott. Analysis and interpretation of data: Stapleton, Walcott. Drafting the article: Stapleton, Walcott. Critically revising the article: all authors. Reviewed submitted version of manuscript: all authors. Approved the final version of the manuscript on behalf of all authors: Stapleton. Statistical analysis: Stapleton, Walcott. Administrative/technical/material support: Butler, Ogilvy. Study supervision: Butler, Ogilvy.

\section{Correspondence}

Christopher J. Stapleton, Massachusetts General Hospital, Department of Neurosurgery, 55 Fruit St., White 502, Boston, MA 02114. email: cstapleton@partners.org. 